\title{
Clusters in a multigraph with elevated density
}

\author{
Mark K. Goldberg* \\ Submitted: Jun 4, 2006; Accepted: Dec 23, 2006; Published: Jan 10, 2007
}

\begin{abstract}
In this paper, we prove that in a multigraph whose density $\Gamma$ exceeds the maximum vertex degree $\Delta$, the collection of minimal clusters (maximally dense sets of vertices) is cycle-free. We also prove that for multigraphs with $\Gamma>\Delta+1$, the size of any cluster is bounded from the above by $(\Gamma-3) /(\Gamma-\Delta-1)$. Finally, we show that two well-known lower bounds for the chromatic index of a multigraph are equal.
\end{abstract}

\section{Introduction}

The chromatic index $\chi^{\prime}(G)$ of a multigraph $G(V, E)$ is the minimal number of colors needed to color all edges of $G$ so that no two edges incident to the same vertex have the same color. A trivial lower bound for $\chi^{\prime}(G)$ is

$$
\Delta(G) \leq \chi^{\prime}(G)
$$

where $\Delta(G)$ is the maximal vertex degree in $G$. A remarkable result discovered by Vizing (see [16]) gives the upper bound $\chi^{\prime}=\chi^{\prime}(G) \leq \Delta(G)+p(G)$, where $p(G)$ is the maximal number of parallel edges in $G$. Thus, for multigraphs without parallel edges (graphs), there are just two possible values for $\chi^{\prime}$ : either $\Delta$, or $\Delta+1$.

For general multigraphs, $p(G) \geq 1$, Shannon proved in [14] that $\chi^{\prime}(G) \leq\lfloor(3 \Delta) / 2\rfloor$, which, taking Vizing's bound into account, is strengthened to $\chi^{\prime} \leq \Delta+\min \{p,\lfloor\Delta / 2\rfloor\}$. The basic question in multigraph edge-coloring is: "what properties of a multigraph cause its chromatic index $\chi^{\prime}$ to exceed $\Delta$ ?" Although to decide if $\chi^{\prime}(G)=\Delta(G)$ is NP-complete, as proved by Hoyler ([8]), it is suspected that for multigraphs with $\chi^{\prime}(G)>\Delta(G)+1$, $\chi^{\prime}(G)$ can be completely characterized in terms of their density $\Gamma(G)$, defined by

$$
\Gamma(G)=\max _{H \subseteq G}\left\lceil\frac{e(H)}{\lfloor v(H) / 2\rfloor}\right\rceil,
$$

${ }^{*}$ Department of Computer Science, Rensselaer Polytechnic Institute, Troy, NY, 12180; E-mail: goldberg@cs.rpi.edu 
where $H$ is a sub-multigraph of $G$ of order at least two and $v(H)$ (resp. $e(H)$ ) denotes the number of vertices (resp. edges) in $H$. It is easy to see that, for every multigraph $G$,

$$
\Gamma(G) \leq \chi^{\prime}(G)
$$

Currently, no multigraph is known with $\chi^{\prime}(G)>\max (\Delta+1, \Gamma)$. Conjectures connecting the chromatic index, maximal degree, and the density of a multigraph were independently proposed by Goldberg ([6]) and Seymour ([13]) more than 25 years ago (see also [9]).

Conjecture 1 (Goldberg ([6]) For every multigraph $G$, if $\chi^{\prime}(G)>\Delta+1$, then $\chi^{\prime}(G)=$ $\Gamma(G)$.

Conjecture 2 (Seymour [13]) For every multigraph $G, \chi(G) \leq \max \{\Delta(G), \Gamma(G)\}+1$.

An extension of the conjectures above was proposed by Goldberg in [7]:

Conjecture 3 If $\Delta \neq \Gamma$, then $\chi^{\prime}=\max \{\Delta, \Gamma\}$, else $\chi^{\prime} \leq \Delta+1$.

Since all three conjectures are closely related to each other, we globally refer to them as the GS-conjecture. See $[2,7,10,11,15,5]$ for some results towards the conjecture; in particular, Nishizeki and Kashiwagi $([11])$ proved $\chi^{\prime}=\Gamma$ for multigraphs with $\chi^{\prime}>$ $(11 \Delta+8) / 10$, and Favrholdt, Steibitz, and Toft, $([5])$ proved $\chi^{\prime}=\Gamma$ for multigraphs with $\chi^{\prime}>(13 \Delta+10) / 12$; the latter is based on the Tashkinov's result from [15].

The GS-conjecture motivates the study of the multigraphs with $\Gamma>\Delta$; we call them multigraphs with elevated density. The properties of the multigraphs with elevated density presented here are formulated in terms of two new notions: set-cycles and multigraph clusters.

Definition 1 A sequence $\mathcal{S}=\left\{S_{i}\right\}_{i=1}^{k}$ of sets is called a set-cycle if

$$
\forall i \in[1, k], S_{i} \cap S_{i+1} \neq \emptyset \& S_{i} \cap S_{i+1} \cap S_{i+2}=\emptyset .
$$

Here $S_{k+1}=S_{1}$ and $S_{k+2}=S_{2}$.

A collection $\mathcal{T}=\left\{T_{j}\right\}_{j=1}^{m}$ is called a set-forest, if no sequence of sets from $\mathcal{T}$ is a set-cycle.

Definition 2 Given a multigraph $G(V, E)$, a set $S \subseteq V$ is called maximally dense, or a cluster, if $e(S)>(\Gamma-1)\lfloor|S| / 2\rfloor$. A cluster $S$ is called minimal if no proper subset of $S$ is a cluster.

Thus, the clusters are subgraphs for which the lower bound for $\chi^{\prime}(G)$ is achieved. Note that the notion of a cluster is close to that of an overfull graph introduced by A.J.W. Hilton. A simple graph is called overfull, if $|E(G)|>\Delta(H)\lfloor|V(H)| / 2\rfloor$. Clearly, if $G$ contains an overfull subgraph $H$ with $\Delta(G)=\Delta(H)$, then $\chi^{\prime}(G)=\Delta(G)+1$. A.J.W. Hilton asked if the reversed is true for graphs with $\Delta(G)>|V(G)| / 3$ (see [3, 4] and [9] for the history of the question). 
Our main result (Section 2) establishes that the collection of minimal clusters in a multigraph with $\Gamma>\Delta$ has a simple structure: it is a set-forest. We also prove that in a multigraph with $\Gamma>\Delta+1$, the size of any cluster is bounded by a function which depends on $\Gamma$ and $\Delta$ only (not on the number of the vertices of the multigraph). This bound matches the upper bound of the size of a critical multigraph which was proved in [6] under the assumption of the GS-conjecture.

A lower bound for $\chi^{\prime}(G)$, which is sometimes stronger than $\Gamma(G)$, can be formulated in terms of maximum matchings of subgraphs of $G$.

Definition 3 Let $F \subseteq E$ and let $m(F)$ denote the maximal size of a matching comprised of edges in F. Then,

$$
\Omega(G)=\max _{F \subseteq E(G)}\left\lceil\frac{|F|}{m(F)}\right\rceil
$$

It is easy to see that

$$
\Gamma(G) \leq \Omega(G) \leq \chi^{\prime}(G) .
$$

A star is an example of a multigraph with $\Gamma(G)<\Omega(G)$. If there were multigraphs with $\Delta \leq \Gamma<\Omega$, the GS-conjecture would be disproved. However, in Section 3, we use Tutte's matching theorem to prove that for every multigraph $G$,

$$
\Omega(G)=\max \{\Delta(G), \Gamma(G)\} .
$$

The notion of $\Omega(G)$ is close to that of the fractional edge chromatic number $\chi_{f}^{\prime}$ introduced by Berge in [1] (see also Chapter 4 in [12]):

Definition 4 A fractional edge coloring of $G$ is an assignment of a non-negative weight $w_{M}$ to each matching $M$ in $G$ so that for every edge $e \in E(G), \sum_{M: e \in M} w_{M} \geq 1$. The fractional edge chromatic number, $\chi_{f}^{\prime}(G)$, is then defined by

$$
\chi_{f}^{\prime}(G)=\min _{M} \sum_{M} w_{M}
$$

Using Edmond's matching polytop theorem, Scheinerman and Ullman ([12]) derived

$$
\chi_{f}^{\prime}(G)=\max \left\{\Delta(G), \max _{H \subseteq G,|V(H)| \geq 2} \frac{e(H)}{\lfloor v(H) / 2\rfloor}\right\}
$$

Thus, $\chi_{f}^{\prime}(G) \leq \Omega(G) \leq \chi^{\prime}(G)$, and for multigraphs with $\chi_{f}^{\prime}>\Delta(G), \Omega(F)=\left\lceil\chi_{f}^{\prime}(G)\right\rceil$. Note that our proof of (3) is significantly simpler than that of (4).

The following notations are used in this paper. Given a set $S \subseteq V(G), G[S]$ denotes the subgraph induced by $S$. If $F \subseteq E(G)$, then $G[F]$ denotes the subgraph of $G$ induced by $F$ : the vertex set of $G[F]$ is the set of vertices incident to the edges in $F$, and the set of edges of $G[F]$ is set $F$. Unless otherwise specified, $\operatorname{deg}(x)$ denotes the degree of a vertex $x$ in $G$; given $S, T \subseteq V(G), \operatorname{deg}(S, T)$ denotes the number of edges $x y$ such that $x \in S$ and $y \in T$; $\operatorname{deg}_{S}(x)$ denotes the degree of $x$ in the subgraph $G[S]$ induced on $S$; $\delta_{S}(x)=\operatorname{deg}(x)-\operatorname{deg}_{G[S]}(x) ; \delta(S)=\sum_{x \in S} \delta_{S}(x)=\operatorname{deg}(S, V(G)-S) ; \quad \nabla(x)=\Delta-\operatorname{deg}(x) ;$ and $\nabla(S)=\sum_{x \in S} \nabla(x)$. See [17] for undefined notations. 


\section{Topology of minimal clusters.}

The goal of this section is to establish several structural properties of the set of minimal clusters in a multigraph $G$ with $\Gamma=\Gamma(G)>\Delta(G)=\Delta$. We also give an upper bound for the size of any cluster in a multigraph with $\Gamma(G)>\Delta+1$; it turns out that for such multigraphs, the cluster size is bounded from the above by a function depending on $\Delta$ and $\Gamma$ only. Throughout this section, $G$ is a multigraph with $\Gamma(G)>\Delta(G)$. If $S \subseteq V(G)$, then $e(S)$ denotes $e(G[S])$. The first lemma is a simple extension of the standard inequality $2 e(S) \leq \Delta|S|$.

Lemma 1 For every subset $S \subseteq V(G)$,

$$
\delta(S)+\nabla(S)+2 e(S)=\Delta|S| .
$$

Proof. The result follows from

$$
\begin{aligned}
2 e(S) & =\sum_{x \in V(S)} \operatorname{deg}_{S}(x)=\sum_{x \in V(S)}\left(\operatorname{deg}(x)-\delta_{S}(x)\right) \\
& =\sum_{x \in V(S)}\left(\Delta-\nabla(x)-\delta_{S}(x)\right) \\
& =|V(S)| \Delta-\nabla(S)-\delta(S) .
\end{aligned}
$$

Lemma 2 The cardinality of every cluster $S$ in $G$ is odd.

Proof. If $|S|$ were even, then the defining inequality $(\Gamma-1)\lfloor|S| / 2\rfloor<e(S)$ could be rewritten as

$$
(\Gamma-1)|S|<2 e(S)
$$

Since $2 e(S) \leq \Delta|S|$, it would imply

$$
(\Gamma-1)|S|<2 e(S) \leq \Delta|S|,
$$

which contradicts our assumption $\Delta<\Gamma$. II

Lemma 3 For every cluster $S$,

$$
\delta(S)+\nabla(S) \leq \Delta-2-(\Gamma-\Delta-1)(|S|-1) .
$$

Proof. Since $|S|$ is odd,

$$
(\Gamma-1)\left\lfloor\frac{|S|}{2}\right\rfloor=(\Gamma-1) \frac{|S|-1}{2}<e(S) .
$$

This implies $(\Gamma-1) \frac{|S|-1}{2}+1 \leq e(S)$, which, in turn, yields

$$
(\Gamma-1)(|S|-1)+2 \leq 2 e(S) \text {. }
$$

Combining the latter with $\Delta|S|-\nabla(S)-\delta(S)=2 e(S)$ (Lemma 1), we have

$$
(\Gamma-1)(|S|-1)+2 \leq \Delta|S|-\nabla(S)-\delta(S),
$$

which is equivalent to (6). II 
Lemma 4 For any two minimal clusters $S_{1}$ and $S_{2}$ of a multigraph $G$, if $S_{1} \cap S_{2} \neq \emptyset$, then $\left|S_{1} \cap S_{2}\right|$ is odd.

Proof. Let us assume that $\left|S_{1} \cap S_{2}\right|=2 a$, where $a$ is a positive integer. Let $A=$ $S_{1} \cap S_{2} ; \quad e_{o}=e(G[A]) ; 2 p_{i}+1=\left|S_{i}\right| ; \quad e_{i}=e\left(G\left[S_{i}-A\right]\right)$, and $w_{i}=\operatorname{deg}\left(A, S_{i}-A\right)$ $(i=1,2)$. By definition of a cluster, $(\Gamma-1) p_{i}<e_{i}+w_{i}+e_{0}(i=1,2)$, implying

$$
(\Gamma-1)\left(p_{1}+p_{2}\right)<e_{1}+e_{2}+w_{1}+w_{2}+2 e_{0} .
$$

Since $\left|S_{i}-A\right|=2 p_{i}-2 a+1$, by the minimality of cluster $S_{i}, e_{i} \leq\left(p_{i}-a\right)(\Gamma-1)(i=1,2)$, hence

$$
e_{1}+e_{2} \leq\left(p_{1}+p_{2}-2 a\right)(\Gamma-1)=\left(p_{1}+p_{2}\right)(\Gamma-1)-2 a(\Gamma-1) .
$$

By Lemma $1, w_{1}+w_{2}+2 e_{0} \leq 2 a \Delta \leq 2 a(\Gamma-1)$. Plugging it into (8), we obtain

$$
e_{1}+e_{2}+w_{1}+w_{2}+2 e_{0} \leq(\Gamma-1)\left(p_{1}+p_{2}\right),
$$

which contradicts inequality (7). II

It is easy to construct examples of minimal clusters that intersect. The multigraph in Figure 1 shows that the intersection of two minimal clusters can have more than one vertex.

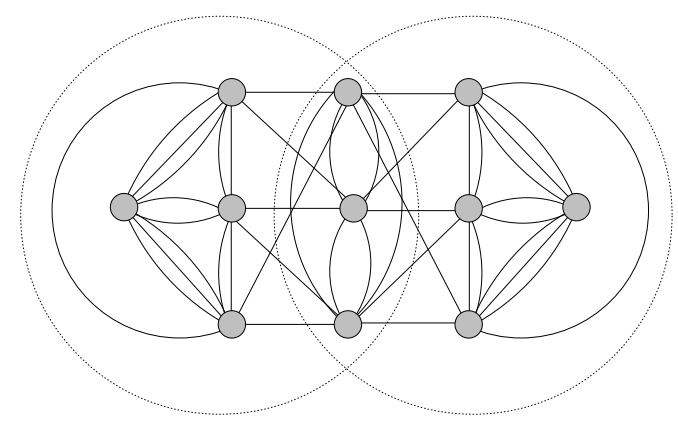

Figure 1: The intersection of two minimal clusters within dotted circles consists of three vertices; for the multigraph, $\Gamma=9$; and $\Delta=8$.

Theorem 1 The set $\mathcal{T}=\left\{S_{i}\right\}_{i=1}^{m}$ of all minimal clusters in a multigraph $G$ is a set-forest.

Proof. Suppose that, contrary to the statement, there is a set-cycle $\left\{S_{i}\right\}_{i=1}^{k}$ all of whose sets are minimal clusters in $G$. Let $A_{i}=S_{i} \cap S_{i-1}$ and $B_{i}=S_{i}-A_{i}-A_{i+1}(i \in[1, k])$. As before, we use indices "cyclically": $A_{1}=S_{1} \cap S_{k}$ and $B_{k}=S_{k}-A_{k}-A_{1}$.

Since $\left|S_{i}\right|$ and $\left|A_{i}\right|$ are odd (Lemmas 2 and 4), and $A_{i} \cap A_{i+1}=\emptyset$ (from the definition of a set-cycle), it follows that $\left|B_{i}\right|=\left|S_{i}\right|-\left|A_{i}\right|-\left|A_{i+1}\right|$ is also odd $(i \in[1, k])$.

Let $\left|A_{i}\right|=2 a_{i}+1,\left|B_{i}\right|=2 b_{i}+1, w_{i}^{+}=\operatorname{deg}\left(A_{i}, S_{i}-A_{i}\right)$, and $w_{i}^{-}=\operatorname{deg}\left(A_{i}, S_{i-1}-A_{i}\right)$ $(i \in[1, k])$. Clearly,

$$
e\left(S_{i}\right) \leq e\left(A_{i}\right)+e\left(B_{i}\right)+e\left(A_{i+1}\right)+w_{i}^{+}+w_{i+1}^{-} .
$$




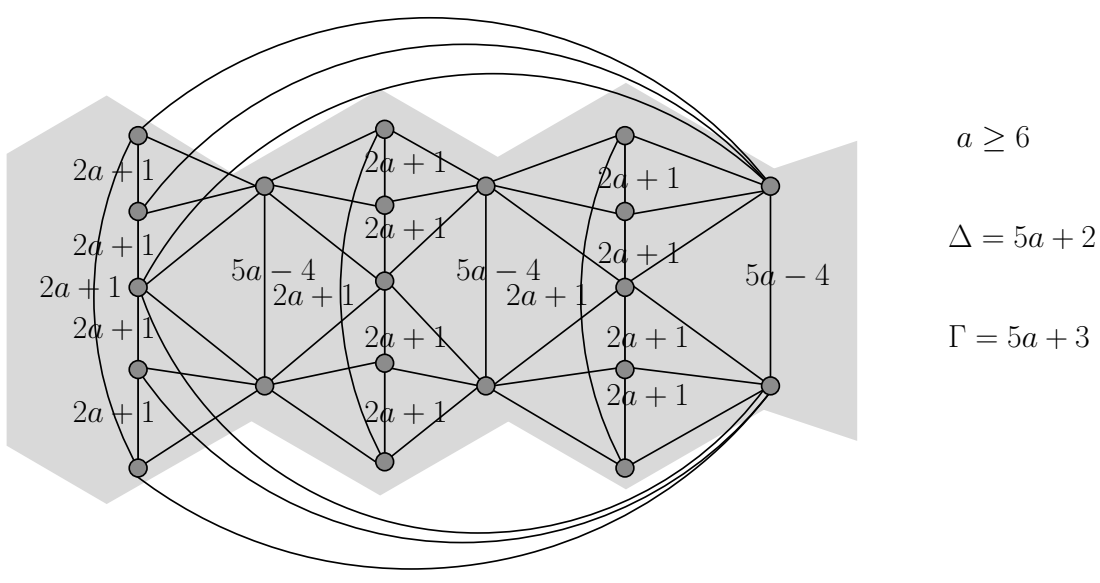

Figure 2: This multigraph has a set-cycle composed of non-minimal clusters. The labels on the edges indicate their multiplicities; the shaded 6-gons indicate clusters; the two right-most vertices belong to two clusters.

Since $A_{i}$ and $B_{i}$ are proper subsets of minimal clusters.

$$
e\left(A_{i}\right) \leq(\Gamma-1) a_{i} \text { and } e\left(B_{i}\right) \leq(\Gamma-1) b_{i}(i \in[1, k]) .
$$

Thus,

$$
\begin{aligned}
\sum_{i=1}^{k} e\left(S_{i}\right) & \leq \sum_{i=1}^{k}\left((\Gamma-1) a_{i}+(\Gamma-1) b_{i}+(\Gamma-1) a_{i+1}\right)+\sum_{i=1}^{k}\left(w_{i}^{+}+w_{i+1}^{-}\right) \\
& =(\Gamma-1) \sum_{i=1}^{k}\left(2 a_{i}+b_{i}\right)+\sum_{i=1}^{k}\left(w_{i}^{-}+w_{i+1}^{+}\right) .
\end{aligned}
$$

Since, $w_{i}^{-}+w_{i+1}^{+} \leq \delta\left(S_{i}\right)$ and, by Lemma $6, \delta\left(S_{i}\right) \leq \Delta-2 \leq \Gamma-1$, after some simplifications we have

$$
\sum_{i=1}^{k} e\left(S_{i}\right) \leq(\Gamma-1) \sum_{i=1}^{k}\left(2 a_{i}+b_{i}+1\right) .
$$

On the other hand, by the definition of clusters,

$$
e\left(S_{i}\right)>(\Gamma-1)\left\lfloor\frac{2 a_{i}+1+2 a_{i+1}+1+2 b_{i}+1}{2}\right\rfloor=(\Gamma-1)\left(a_{i}+a_{i+1}+b_{i}+1\right),
$$

which implies

$$
(\Gamma-1) \sum_{i=1}^{k}\left(2 a_{i}+b_{i}+1\right)<\sum_{i=1}^{k} e\left(S_{i}\right) .
$$

Inequality (10) contradicts inequality (9), proving the correctness of the theorem. 
Theorem 2 For every cluster $S$ of a multigraph with $\Gamma>\Delta+1$,

$$
|S| \leq \frac{\Gamma-3}{\Gamma-1-\Delta}
$$

Proof. Express inequality (6) with respect to $|S|$ and then use $0 \leq \delta(S)+\nabla(S)$.

\section{Lower bounds}

Although, for some multigraphs, $\Omega$ is a stronger lower bound for $\chi^{\prime}$ than $\Gamma$, it turns out that is it not stronger than $\Delta$ and $\Gamma$ combined.

Lemma 5 For any multigraph $G$,

$$
\max (\Gamma(G), \Delta(G)) \leq \Omega(G) \leq \chi^{\prime}(G)
$$

Proof. For any edge-coloring of $G$ and any $F \subseteq E(G)$, the number of edges colored the same color does not exceed $m(H) \leq\lfloor\mid V(H) / 2\rfloor$, where $H=G[F]$. Hence,

$$
\chi^{\prime}(H) \geq\left\lceil\frac{|F|}{m(F)}\right\rceil \geq\left\lceil\frac{e(H)}{\left\lfloor\frac{\mid V(H)}{2}\right\rfloor}\right\rceil .
$$

To complete the proof, notice that if $x$ is a vertex of the maximal degree in $G$ and $F$ is the set of edges incident to $x$, then $m(F)=1$, implying that $\Delta(G) \leq \Omega(G)$.

Theorem 3 For every multigraph $G, \max (\Gamma(G), \Delta(G))=\Omega(G)$.

Proof. By Lemma 5, we only need to prove that $\max (\Gamma(G), \Delta(G)) \geq \Omega(G)$. Let $F$ be a set of edges for which

$$
\left\lceil\frac{|F|}{m(F)}\right\rceil=\Omega(G)
$$

and let $H=G[F]$. If $m(F) \geq(|V(H)|-1) / 2$, the result follows immediately. Thus, we assume that

$$
m(F)<\frac{|V(H)|-1}{2} \text { and } \Omega>\max (\Delta, \Gamma) .
$$

By Tutte's theorem ([17]), there is a subset $K \subseteq V(H)$ such that the number of odd connected components of $H-K$ is $q=k+n-2 m(F)$, where $n=|V(H)|$ and $k=|K|$.

Let $\left\{V_{i}, F_{i}\right\}_{i=1}^{q+t}$ be the connected components of $G-K$, where the first $q$ of them are odd and the remaining $t$ are even. Let $\left|V_{i}\right|=2 a_{i}+1$, for $i \in[1, q]$ and $\left|V_{i}\right|=2 a_{i}$, for $i \in[q+1, q+t]$. Let $C_{i}$ be the set of edges of $H$ with one endpoint in $V_{i}$ and the other in $K(i \in[1, q+t])$. Finally, let $E(K)$ denote the set of edges in $F$ with both end-points in $K$.

Using the assumption $\Omega>\max (\Delta, \Gamma)$, for every odd connected component,

$$
\forall i \in[1, q], \quad\left|F_{i}\right| \leq \Gamma a_{i} \leq(\Omega-1) a_{i}
$$




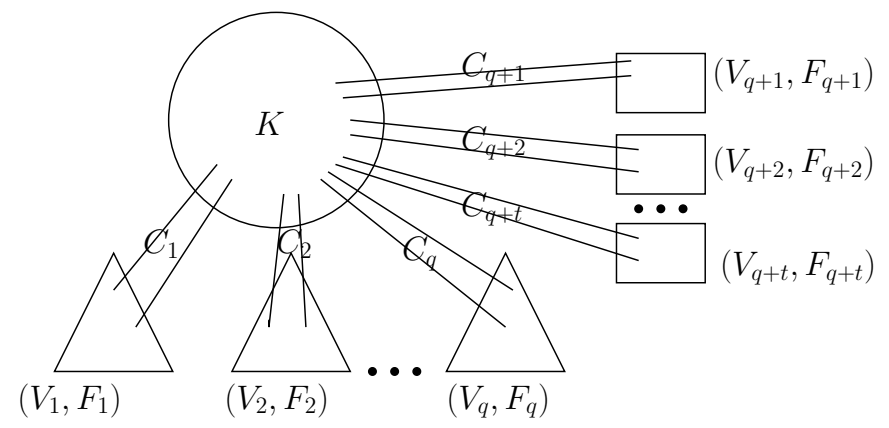

Figure 3: The triangles (resp. squares) represent odd (resp. even) components.

Since the maximum vertex degree is $\Delta$,

$$
\begin{gathered}
\forall i \in[q+1, q+t], \quad\left|F_{i}\right| \leq \Delta a_{i} \\
|E(K)|+\sum_{i=1}^{q+t}\left|C_{i}\right| \leq 2|E(K)|+\sum_{i=1}^{q+t}\left|C_{i}\right| \leq \Delta k .
\end{gathered}
$$

Combining (12), (13), (14), and using $\Omega-1 \geq \Delta$ one more time, we get an upper bound for $|F|$ :

$$
|F| \leq|E(K)|+\sum_{i=1}^{q+t}\left(\left|F_{i}\right|+\left|C_{i}\right|\right) \leq \Delta k+(\Omega-1) \sum_{i=1}^{q} a_{i}+\Delta \sum_{i=q+1}^{q+t} a_{i} \leq(\Omega-1)\left(k+\sum_{i=1}^{q+t} a_{i}\right) .
$$

Since

$$
n=\sum_{i=1}^{q}\left(2 a_{i}+1\right)+\sum_{i=q+1}^{q+t}\left(2 a_{i}\right)+k
$$

we have

$$
m(F)=\sum_{i=1}^{q+t} a_{i}+k .
$$

Using this expression for $m(F)$ and inequality (15),

$$
\Omega=\left\lceil\frac{|F|}{m(F)}\right\rceil \leq \frac{(\Omega-1)\left(\sum_{i=1}^{q+t} a_{i}+k\right)}{\sum_{i=1}^{q+t} a_{i}+k}=\Omega-1 .
$$

The contradiction disproves the assumption and completes the proof.

Acknowledgment: The author is grateful to Bjarne Toft for pointing out the connection of Theorem 3 and the results in fractial edge-coloring; and to the anonymous reviewer for many suggestions that improved the exposition of the paper. The work presented in this paper is partially supported by the National Science Foundation under Grant No. 0324947. Any opinions, findings, and conclusions or recommendations expressed in this material are those of the author and do not necessarily reflect the views of the National Science Foundation. 


\section{References}

[1] C. Berge. Fractional Graph Theory. ISI Lecture Notes 1, Macmillan of India, 1978.

[2] D. Cariolaro. On fans in multigraphs. Journal of Graph Theory, 51(4):301-318, 2006.

[3] A. Chetwynd and A. Hilton. Star multigraphs with three vertices of maximum degree. Math. Proc. Cambridge Philos. Soc., 100:303-317, 1986.

[4] A. Chetwynd and A. Hilton. The edge-chromatic class of graphs with maximum degree at least $|v|-3$. in: L.D. Andersen, I.T.Jakobsen, C. Thomassen, B. Toft, and P.D. Vestergaard, editors, Graph Theory in Memory of G. A. Dirac, Annals of Discrete Mathematics, 100:303-317, 1989.

[5] L. Favrholdt, M. Steibitz, and B. Toft. Graph colouring: Vizing's Theorem and Goldberg's Conjecture. IMADA preprint: PP - 2006-20, 2006.

[6] M. K. Goldberg. On multigraphs of almost maximal chromatic class. Discret. Analiz, 23:3-7, 1973. In Russian.

[7] M. K. Goldberg. Edge coloring of multigraphs: Recoloring technique. J. Graph Theory, 8:123-137, 1984.

[8] I. Holyer. The NP-completeness of edge coloring. SIAM J. Comput., 10:718-720, 1981.

[9] T. Jensen and B. Toft. Graph coloring problems. Wiley-Interscience Publications, John Wiley 85 Sons, 1995.

[10] L. Kahn. Asymptotics of the chromatic index for multigraphs. J. Combin. Theory Ser. B, 68:195-235, 1996.

[11] T. Nishizeki and K. Kashiwagi. On the 1.1 edge-coloring of multigraphs. SIAM J. Discrete Math., 3:391-410, 1990.

[12] E. R. Scheinerman and D. H. Ullman. Fractional Graph Theory: A Rational Approach to the Theory of Graphs. Wiley Interscience, New York, 1997.

[13] P. D. Seymour. Some unsolved problems on one-factorizations of graphs. Graph Theory and Related Topics, Academic Press, 1979. Bondy and Murty, eds.

[14] C. E. Shannon. A theorem on coloring the lines of a network. J. Math. Phys., 28:148-151, 1949.

[15] V. A. Tashkinov. On an algorithm for the edge coloring of multigraphs. Discretn. Anal. Issled. Oper., 1(7):72-85, 2000. in Russian.

[16] V. G. Vizing. Critical graphs with a given chromatic class. Discret. Analiz, 5:9-17, 1965. in Russian.

[17] D. B. West. Introduction to graph theory. Prentice Hall, Upper Saddle River, NJ, 2003. 